\title{
Qualitative similarities in the visual short-term memory of pigeons and people
}

\author{
Brett Gibson • Edward Wasserman • Steven J. Luck
}

Published online: 12 July 2011

(C) Psychonomic Society, Inc. 2011

\begin{abstract}
Visual short-term memory plays a key role in guiding behavior, and individual differences in visual shortterm memory capacity are strongly predictive of higher cognitive abilities. To provide a broader evolutionary context for understanding this memory system, we directly compared the behavior of pigeons and humans on a change detection task. Although pigeons had a lower storage capacity and a higher lapse rate than humans, both species stored multiple items in short-term memory and conformed to the same basic performance model. Thus, despite their very different evolutionary histories and neural architectures, pigeons and humans have functionally similar visual short-term memory systems, suggesting that the functional properties of visual short-term memory are subject to similar selective pressures across these distant species.
\end{abstract}

Keywords Animal cognition · Comparative cognition . Comparative psychology $\cdot$ Visual working memory

Electronic supplementary material The online version of this article (doi:10.3758/s13423-011-0132-7) contains supplementary material, which is available to authorized users.

\section{B. Gibson $(\bowtie)$}

Department of Psychology, University of New Hampshire,

Conant Hall, Durham,

NH 03824-3567, USA

e-mail: bgibson@cisunix.unh.edu

E. Wasserman

Department of Psychology, University of Iowa, Iowa City, IA, USA

\section{S. J. Luck}

Department of Psychology, University of California, Davis, CA, USA
In The Descent of Man, Charles Darwin stated that “. . . the difference in mind between man and the higher animals, great as it is, is certainly one of degree and not of kind" (1871/2006, p. 837). One mental process that may be particularly well conserved across species is visual shortterm memory (VSTM). Although our visual world appears to involve a continuous flow of information, our perceptions are actually separated by eye movements and blinks. VSTM plays an essential role in daily functioning by allowing us to integrate these punctuated views into a single representation. Comparing objects in VSTM with those currently in view is also critical to comparing objects that cannot be perceived simultaneously. VSTM capacity develops rapidly during infancy (Oakes, Ross-Sheehy, \& Luck, 2006; Rose, Feldman, \& Jankowski, 2004), is mediated by the posterior parietal cortex (Todd \& Marois, 2004), and relies on neural synchrony (Siegel, Warden, \& Miller, 2009).

A key element of human VSTM is the ability to maintain multiple objects concurrently in memory. Very simple recurrent circuitry is sufficient to retain a single item (Deco \& Rolls, 2008), but retaining multiple items without confusing them is a much more difficult computational problem (Luck, 2008). Moreover, individual differences in human VSTM capacity are strongly correlated with higher cognitive abilities, explaining over $40 \%$ of the variance in global fluid intelligence (Fukuda, Vogel, Mayr, \& Awh, 2010) and almost $80 \%$ of the variance in cognitive task performance (Gold et al., 2010) among healthy adults.

The ability to integrate across eye movements and to compare objects should have strong adaptive value not only for people, but for nonhuman animals; after all, they too must detect predators or prey, choose mates, and 
determine where to forage (see Shettleworth, 2010, and Wasserman \& Zentall, 2006, for summaries). Given that VSTM should be valuable for a wide range of animal species, it is important to determine whether organisms with very different evolutionary histories and neural architectures have functionally similar VSTM systems. That is, do they differ in degree rather than kind, as Darwin suggested?

One species that is well suited for comparing with people in terms of VSTM is the pigeon; the pigeon too has a highly developed visual system, but it relies on a highly developed tectum and nonlaminated pallial structures rather than a laminated neocortex (Granda \& Maxwell, 1979; Shimizu, 2009). Thus, the basic computational machinery of the pigeon visual system is very different from that of the human visual system, even though both arose from common structures over evolution.

Recent work using change detection procedures developed for people has suggested that pigeons can detect changes in visual scenes (Lazareva, Wasserman, \& Luck, 2006; Wright et al., 2010), but it is not yet clear whether they can maintain multiple items concurrently in VSTM. Given that the parietal cortex plays a key role in human VSTM capacity (Todd \& Marois, 2004), but that the pigeon entirely lacks a layered neocortex, it may be the case that pigeons utilize a more primitive representation in which multiple items cannot be maintained concurrently. That is, they may have a capacity of only one item.

To examine this issue, we designed a change detection task $^{1}$ with three prime properties. First, because we do not yet know what visual features are stored in pigeon VSTM, we used complex objects that changed along multiple dimensions. Second, performance in typical change detection tasks declines as the set size increases; pigeons may learn that the probability of reward declines as the set size increases, causing motivation to be correlated with set size. To avoid this problem, we maintained a constant set size and unpredictably varied the number of items that changed. Consequently, the participants could not anticipate the difficulty of the task for a given trial until the very end of the trial. Third, we included trials on which all of the items in the display changed. An observer with even the most rudimentary VSTM abilities

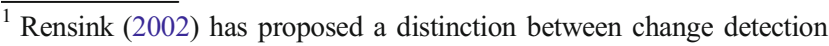
and difference detection. Change detection implies that the observer has detected a change in a given set of objects over time, whereas difference detection merely implies that two stimulus arrays are different. At present, we have no way of determining whether pigeons perceive differences between sequential displays as changes to the same underlying structure or simply as differences between completely distinct displays. However, both change detection and difference detection would require VSTM in the present task; we use the phrase "change detection task" simply because this is the common description for this type of task.
}

should be $100 \%$ correct on these trials, and errors on these trials should reflect nonspecific factors such as lapses of attention or response confusions.

We used a quantitative model to separately estimate VSTM storage capacity $(K)$ and the rate of nonspecific lapses $(L)$ from the pattern of data. Roughly speaking, the lapse rate is related to the asymptotic error rate when all items change. Capacity is determined from the likelihood that a change will be detected if an observer can store $K$ items in VSTM; if one of the eight items in the display changes, then there is a $K / 8$ chance that the changed item will be one of the $K$ items in memory (Pashler, 1988). It is necessary to account for the lapse rate and for guessing in computing $K$, but methods have recently been developed for doing so in the human VSTM literature (Rouder, Morey, Morey, \& Cowan 2011). It is important to note that this model assumes that observers store high-quality representations of all $K$ items and have no information about the remaining items (which appears to be true in humans; see Anderson, Vogel, \& Awh 2011; Zhang \& Luck, 2008). However, it is possible to avoid this assumption by treating $K$ as the number of items' worth of information stored in VSTM (Vogel, Woodman, \& Luck, 2001).

\section{Method}

\section{Participants}

We tested 30 undergraduate students (18 females, 12 males; ages 18 to 27 years) who had normal or corrected-to-normal vision. The participants gave informed consent prior to participating in the study, and they received course credit for arriving at the experimental session. Three feral pigeons were also tested. They were housed in individual cages and were food restricted to $85 \%$ of their ad lib weights using controlled feedings of mixed grain following experimental sessions.

\section{Procedure}

Each pigeon was trained and tested in a $36(\mathrm{~h}) \times 36(\mathrm{l}) \times 41$ (w) $\mathrm{cm}$ operant chamber. Visual images were presented on an LCD computer monitor (NEC Model 1530V), and a touchscreen (Model E14603-000; Elotouch, Fremont, CA) recorded the location of responses (pecks) that the birds made to the screen. A rotary feeder that was controlled by a Macintosh computer dispensed food pellets (45 mg apiece; Research Diets, Inc., New Brunswick, NJ) into a food cup inside the chamber following correct responses.

Training with change and no-change displays began after the initial period of shaping to peck geometric shapes on the screen. A start stimulus - a black cross - appeared in 
the center of a $10 \times 12 \mathrm{~cm}$ display area that was centered on the monitor to start a trial. The display area was colored white, whereas the remainder of the screen was a black background. A peck to the start stimulus advanced the trial. An array of 8 images (each approximately $1.5 \times 1.5 \mathrm{~cm}$ ) then appeared within the display area (Fig. 1), each of which was a colored line drawing of a common object. The 8 images were randomly selected without replacement from a pool of 16 unique images. Each of the 8 images was randomly placed into 1 of 12 cells, which formed a virtual $3 \times 4$ grid $(2.5 \mathrm{~cm}$ center-to-center spacing) centered within the display area. The images that were used to construct the displays were balanced across trials. The display was presented for $1 \mathrm{~s}$ and then disappeared. Following a 1-s interstimulus interval (ISI), a second display of 8 images (test display) then appeared on the screen for $1 \mathrm{~s}$. The locations of the images on the screen were the same for both the sample and test displays on a given trial.

Throughout training, change and no-change trials were randomly intermixed with equal probabilities, with the constraint that no more than three consecutive trials of either type could occur. On no-change trials, the identities of the all of the images in the arrays were the same for the sample and test displays. On change trials during the training phase, the identities of all of the images were different between the sample and test displays; that is, 8 of the 16 images in the pool were randomly selected to create the sample display, and the remaining 8 images were used to create the test display.

After the test display disappeared, red and green report keys $(2 \times 6 \mathrm{~cm}$ colored rectangles on the monitor) immediately appeared $1 \mathrm{~cm}$ to the left and to the right of the display area, respectively (see Fig. 1). A correct response resulted in the delivery of two food pellets, followed by a brief intertrial interval $(15 \mathrm{~s})$ and the start of the next trial. An incorrect response resulted in the entire screen turning black and the houselight being turned off for $20 \mathrm{~s}$; a correction trial then ensued. Correction trials were not scored, but they continued until a correct response was made. Each daily session of training consisted of eight

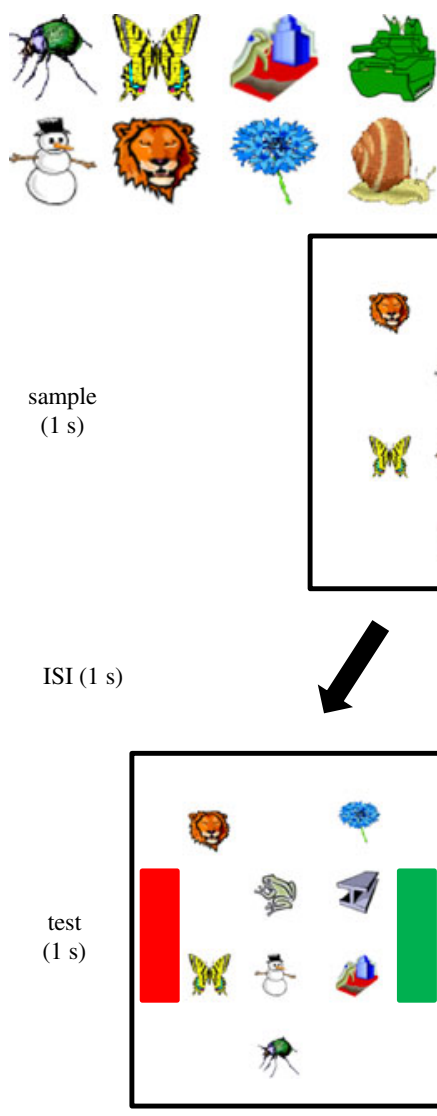

no change condition
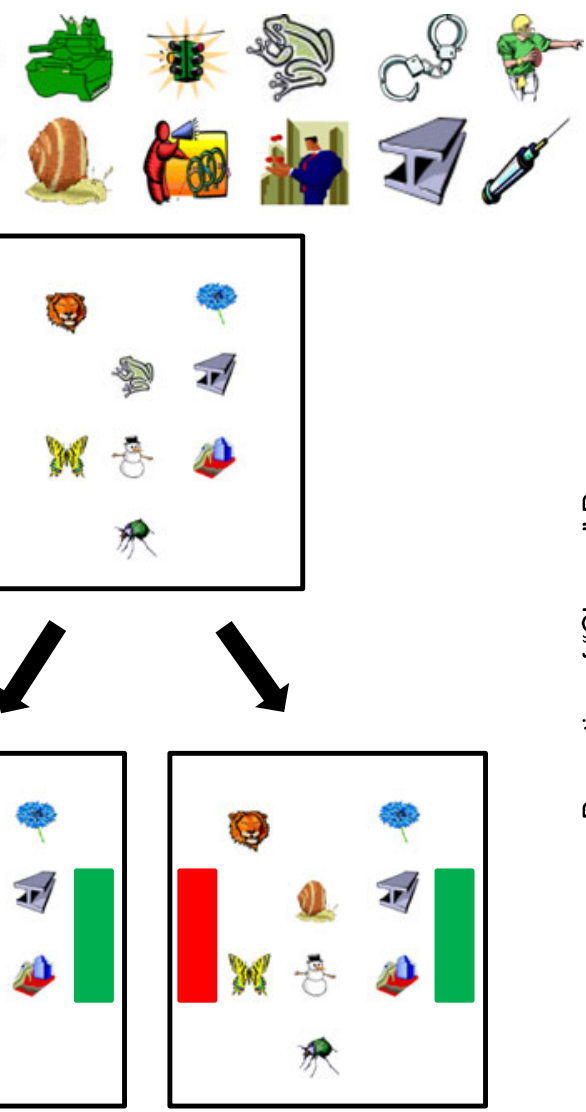

change condition

Fig. 1 (Left) Stimulus set and examples of sample and test stimuli on no-change and change trials. The choice buttons (to the left and right of the display) did not appear until after the stimulus disappeared, but they are shown in the same display here for convenience. (Right)

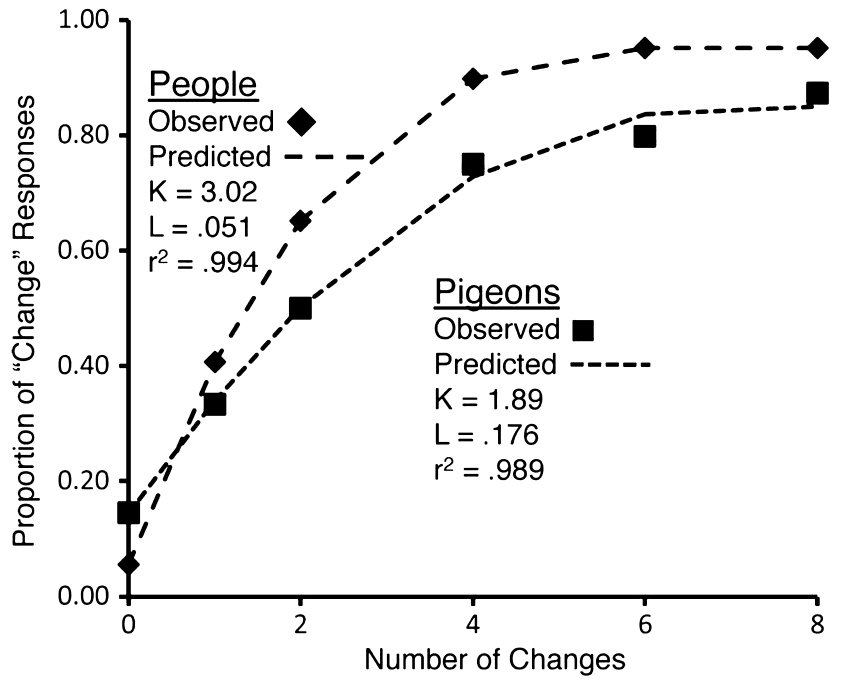

Probability of a change response as a function of the number of changes in the display for pigeons and people. The filled symbols show the observed values, whereas the curves show the estimates derived from model fitting 
blocks; each block contained 8 no-change trials and 8 change trials in randomized order. Training continued until a bird made $85 \%$ correct responses to both the change trials and the no-change trials in a session. The 3 birds took 25, 32, and 58 days of training (128 trials/day), respectively, to reach criterion.

In the testing phase, the goal was to examine change detection performance as the number of changed items varied. That is, the total number of items in the display was held constant at eight, but between one and eight of these items could change between the sample and test displays of a given trial (see Fig. 1). However, to ensure that the association between the classes of trials (change and no-change) and the two response keys did not deteriorate due to large numbers of incorrect responses, most of the trials within each daily session were training trials, with either zero changes or eight changes. Specifically, each daily session of testing contained 149 trials: 64 no-change trials, 64 change trials on which all eight items changed, and 21 change test trials, in which between one and seven of the eight items changed. Noncontingent food reinforcement followed all responses on trials with one to seven changes; correction trials were not used. If performance on the 128 trials with zero or eight changes dropped below the $85 \%$ criterion, then training resumed in subsequent daily sessions until performance again rose above criterion. Testing continued until the pigeons completed 16 testing sessions.

Human participants were seated in a chair and viewed the stimuli on a 15-in. LCD monitor (the same monitor used with the pigeons) at a viewing distance of approximately $50 \mathrm{~cm}$. The stimuli were identical to those used for the pigeons, except that the number of changed items was $1,2,4,6$, or 8 (with equal probability). Participants completed a single session consisting of 50 change trials and 50 no-change trials.

\section{Analysis}

To quantify VSTM capacity $(K)$, we used a generalization of the standard model for estimating capacity from change detection performance (Pashler, 1988; Rouder et al., 2011). This generalization allowed for multiple changes on each trial instead of assuming a single change. It also included a lapse rate parameter $(L)$, under the assumption that participants would occasionally fail to attend to the sample array or confuse the two response keys, leading to random guessing with a probability of $L$, independent of the number of changes (see the supplemental materials for details of the model). All analyses were limited to trials with $0,1,2,4,6$, or 8 changes, which were tested in both species.

\section{Results}

In the present task, the observer is presented with a display which is followed by a brief offset. Presumably, the human or pigeon observer retains a certain number of bound objects $(k)$ in visual memory slots during the offset between the first and second stimulus presentations. On a change trial, if one of the items that changed $(n)$ was one that was retained in memory, then the participant should report that a change has occurred when the display reappears, thereby making the probability that a change will be reported equal to $k / n$. Thus, performance should be worse when fewer items in the display change and better when more items change. Observers with poorer memory should show reduced performance across all levels of the change condition, but still show improvements with increasing numbers of items changing in the display. Figure 1 (right) shows the mean $k$ score estimates for both people and pigeons across the different display size conditions. Both people and pigeons were more likely to report a change as the number of changes increased from 0 to 8 , suggesting similar roles of memory for both species.

Our generalization of the standard change detection model accounted extremely well for the performance of both people and pigeons, explaining over $99 \%$ of the variance (Pearson's $r^{2}$ ) in the group averages. The model also explained between $96 \%$ and $99 \%$ of the variance in the data from each of the 3 pigeons. The number of trials was much smaller for each human participant than for each pigeon, so the fits were weak for a few people, but the model explained more than $90 \%$ of the variance for 25 of the 28 human participants.

The group-level modeling indicated that the numbers of items retained in VSTM were 2.22 for the pigeons and 3.14 for the humans, with lapse rates of .176 and .063, respectively. The individual-level modeling indicated that the 3 pigeons stored 1.86, 1.99, and 2.99 items in memory, with corresponding lapse rates of .132, .128, and .253. A one-sample $t$ test indicated that mean capacity was significantly greater than 1 for both pigeons $[t(2)=3.586$, $p=.0384]$ and people $[t(27)=7.538, p<.0001]$. The individual-participant models for the humans were more variable, presumably owing to the small number of trials per participant, with a range of 1.6 to 5.9 for the number of items stored and .000 to .140 for the lapse rate (excluding 3 participants for whom the model explained less than $75 \%$ of the variance). The differences between species were significant in heteroscedastic $t$ tests for both capacity $(p<.05)$ and lapse rate $(p<.05)$. Thus, the performance of people and pigeons was qualitatively similar, conforming to the same underlying model of performance with a VSTM capacity exceeding 1, but human VSTM appeared quantitatively 
superior to pigeon VSTM, with a greater storage capacity and a lower lapse rate (see the Discussion section below).

\section{Discussion}

The work presented here is the first to directly compare VSTM in a nonhuman animal and in people. For people, the posterior parietal cortex plays a key role in VSTM capacity, whereas pigeon vision relies mainly on subcortical structures and a nonlaminated pallium. Despite differences in the evolutionary histories and neural architectures of avian and mammalian species, the same model of VSTM accounted extremely well for the behavior of both species. Moreover, pigeon VSTM capacity was significantly greater than one item, suggesting that the avian brain has solved the computational problem of maintaining multiple representations concurrently in VSTM. It is possible that further research will reveal that these similarities are accompanied by differences in the operation of VSTM across pigeons and people, reflecting their different ecological niches. Nonetheless, the fundamental similarities observed here suggest that common selective pressures have guided the evolution of VSTM in pigeons and people. Additional comparative work is needed to determine whether these similarities reflect conservation of a VSTM mechanism from a common ancestor or whether VSTM arose independently in the avian and mammalian lines.

It should be noted that the present procedure may actually underestimate VSTM capacity in pigeons. Specifically, the sample display appeared for 1,000 ms immediately following a peck to the screen, and it is possible that the pigeons' heads were not optimally positioned to perceive all eight items in the array. This constraint makes the observed capacity of approximately two objects even more impressive. It is also possible, however, that human VSTM capacity was also underestimated because they did not have as much practice as the pigeons. However, the main findings did not concern the absolute capacity levels of the two species, but the facts that both had a capacity that exceeded 1.0 and that both conformed to the same underlying performance model.

The excellent fits of the same performance model to both the pigeon and human data suggest that VSTM is functionally similar across species. However, a good fit between a model and a data set does not guarantee that the model is correct (Roberts \& Pashler, 2000); it is possible that other models of change detection could fit the pigeon data. We can, however, rule out models that would predict linear or positively accelerated functions relating the probability of a change response to the number of changes in the display. We can also rule out most models that do not include a lapse rate. And, most importantly, we can rule out models in which information from only a single item is present in VSTM. Thus, even though humans and pigeons may differ in the details of how they detect changes in sequential displays, the present results demonstrate substantial similarities across species in the functioning of VSTM.

Several parameters could be explored in future research to determine further similarities or differences between human and pigeon VSTM systems. First, human VSTM remains stable with retention periods of up to $4-5 \mathrm{~s}$, and then the representations suddenly begin to terminate (Vogel et al., 2001; Zhang \& Luck, 2009); it would be valuable to determine whether pigeon VSTM exhibits a similar time course. This possibility would suggest either that the same underlying mechanism is used across species or that the VSTM system has evolved to meet similar functional demands across species. Second, the present study used a fixed set of 16 objects; it is possible that familiarity influences VSTM performance in pigeons, as it sometimes does in humans (Jackson \& Raymond, 2008; but see Olson \& Jiang, 2004; Pashler, 1988). Future work testing novel sets of 16 items would be useful in this regard. Third, humans appear to store discrete, fixed-resolution representations of the individual items in an array (Anderson et al., 2011; Zhang \& Luck, 2008); it would be useful to determine whether this is also true of pigeons.

Author Note This research was made possible by NIH Grants MH47313 and EY019781 to E.A.W. and NIH Grant MH076226 to S. J.L.

\section{References}

Anderson, D. E., Vogel, E. K., \& Awh, E. (2011). Precision in visual working memory reaches a stable plateau when individual item limits are exceeded. Journal of Neuroscience, 31, 1128-1138.

Darwin, C. L. (2006). In E. O. Wilson (Ed.), From so simple a beginning: The four great books of Charles Darwin. New YorK: Norton. Original work published in 1871.

Deco, G., \& Rolls, E. T. (2008). Neural mechanisms of visual memory: A neurocomputational perspective. In S. J. Luck \& A. Hollingworth (Eds.), Visual memory (pp. 247-289). New York: Oxford University Press.

Fukuda, K., Vogel, E., Mayr, U., \& Awh, E. (2010). Quantity, not quality: The relationship between fluid intelligence and working memory capacity. Psychonomic Bulletin \& Review, 17, 673-679.

Gold, J. M., Hahn, B., Zhang, W., Robinson, B. M., Kappenman, E. S., Beck, V. M., et al. (2010). Reduced capacity but spared precision and maintenance of working memory representations in schizophrenia. Archives of General Psychiatry, 67, 570-577.

Granda, A. M., \& Maxwell, G. H. (1979). Neural mechanisms of behavior in the pigeon. New York: Plenum.

Jackson, M. C., \& Raymond, J. E. (2008). Familiarity enhances visual working memory capacity for faces. Journal of Experimental 
Psychology: Human Perception and Performance, 34, 556568.

Lazareva, O., Wasserman, E. A., \& Luck, S. J. (2006). Change detection in pigeons: Stimulus attributes and binding [Abstract]. Journal of Vision, 6(6), 605a.

Luck, S. J. (2008). Visual short-term memory. In S. J. Luck \& A. Hollingworth (Eds.), Visual memory (pp. 43-85). New York: Oxford University Press.

Oakes, L. M., Ross-Sheehy, S., \& Luck, S. J. (2006). Rapid development of feature binding in visual short-term memory. Psychological Science, 17, 781-787.

Olson, I. R., \& Jiang, Y. (2004). Visual short-term memory is not improved by training. Memory \& Cognition, 32, 1326-1332.

Pashler, H. (1988). Familiarity and visual change detection. Perception \& Psychophysics, 44, 369-378.

Rensink, R. A. (2002). Change detection. Annual Review of Psychology, 53, 245-277. doi:10.1146/annurev.psych.53.100901.135125

Roberts, S., \& Pashler, H. (2000). How persuasive is a good fit. A comment on theory testing. Psychological Review, 107, 358-367.

Rose, S. A., Feldman, J. F., \& Jankowski, J. J. (2004). Infant visual recognition memory. Developmental Review, 24, 74-100.

Rouder, J. N., Morey, R. D., Morey, C. C., \& Cowan, N. (2011). How to measure working memory capacity in the change detection paradigm. Psychonomic Bulletin \& Review, 18, 324-330.

Shettleworth, S. J. (2010). Cognition, evolution, and behavior (2nd ed.). Oxford University Press.
Shimizu, T. (2009). Why can birds be so smart. Background, significance, and implications of the revised view of the avian brain. Comparative Cognition and Behavior Reviews, 4, 103115 .

Siegel, M., Warden, M. R., \& Miller, E. K. (2009). Phasedependent neuronal coding of objects in short-term memory. Proceedings of the National Academy of Sciences, 106, 2134121346.

Todd, J. J., \& Marois, R. (2004). Capacity limit of visual short-term memory in human posterior parietal cortex. Nature, 428, 751754. doi: 10.1038 /nature 02466

Vogel, E. K., Woodman, G. F., \& Luck, S. J. (2001). Storage of features, conjunctions, and objects in visual working memory. Journal of Experimental Psychology: Human Perception and Performance, 27, 92-114.

Wasserman, E. A., \& Zentall, T. R. (2006). Comparative cognition: Experimental explorations of animal intelligence. New York: Oxford University Press.

Wright, A. A., Katz, J., Magnotti, J., Elmore, L. C., Babb, S., \& Alwin, S. (2010). Testing pigeon memory in a change detection task. Psychonomic Bulletin \& Review, 17, 243-249.

Zhang, W., \& Luck, S. J. (2008). Discrete fixed-resolution representations in visual working memory. Nature, 453, 233235. doi: 10.1038 /nature 06860

Zhang, W., \& Luck, S. J. (2009). Sudden death and gradual decay in visual working memory. Psychological Science, 20, 423-428. 